\section{Importancia y relevancia de la ética en la investigación}

\section{Importance and relevance of ethics in research}

\section{Yadira Elizabeth Elizalde Rivera}

Estudiante, Escuela Superior Politécnica de

Chimborazo, Riobamba, Ecuador, yadira.elizalde@espoch.edu.ec,

https://orcid.org/oooo-0003-3856-7515

\section{Cristina Elizabeth Toapanta Cherrez}

Estudiante, Escuela Superior Politécnica de

Chimborazo, Riobamba, Ecuador,

cristina.toapanta@espoch.edu.ec,

https://orcid.org/oooo-0002-1439-1140

\section{Juan Carlos Pomaquero Yuquilema}

Master en Políticas Públicas y Sociales, Escuela

Superior Politécnica de Chimborazo, Riobamba, Ecuador,jpomaquero@espoch.edu.ec,

https://orcid.org/oooo-0003-0952-943X

\section{Resumen}

En el presente artículo científico se analiza la relación entre la Investigación y la Ética y, consecuentemente la importancia de aplicación que representan los principios éticos al momento de realizar una investigación en cualquier rama o materia de la Ciencia.

Este estudio comprende una revisión bibliográfica en base a las definiciones de distintos autores tanto de libros como de revistas y otras fuentes confiables. Seguido de definiciones nos enfocaremos en la importancia de la ética y eso será en la parte del

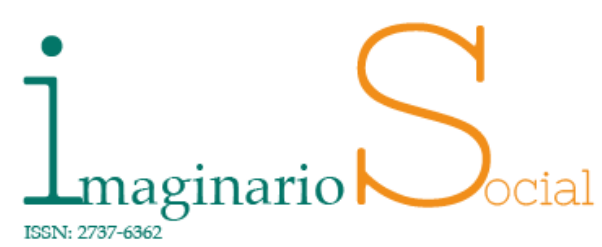

Imaginario Social e-ISSN: 2737-6362

julio - diciembre 2020 Vol. 3-2-2020 http://revista-

imaginariosocial.com/index.php/es/in dex

Recepción: 23 de abril 2019

Aceptación: 15 de febrero 2020

40-51

Atribución/Reconocimiento-NoCo mercial- Compartirlgual 4.0 Licencia Pública Internacional - CC

BY-NC-SA 4.0

https://creativecommons.org/licenses /by-nc- sa/4.0/legalcode.es 
desarrollo del artículo y finalmente concluiremos con que la ética es vital en toda investigación y así podremos permitir que otras personas utilicen nuestra información para futuros textos.

Palabras clave: ética, investigación, técnicas, información científica, ciencia.

\begin{abstract}
This scientific article analyses the relationship between Research and Ethics and, consequently, the importance of applying ethical principles when carrying out research in any branch or subject of Science. This study includes a bibliographic review based on the definitions of different authors from books, magazines and other reliable sources. Followed by definitions we will focus on the importance of ethics and that will be in the development part of the article and finally we will conclude that ethics is vital in all research and so we can allow other people to use our information for future texts.
\end{abstract}

Key word: ethics, research, techniques, scientific information, science.

\title{
Introducción
}

Los avances científicos, empresariales, tecnológicos, políticos, entre otros avanzan a pasos gigantescos, sin retroceso alguno, esto se debe a las múltiples investigaciones que se realizan día a día en el mundo respecto de cada inquietud o problema actual, lo manifestado nos hace pensar que la investigación es eficiente, productiva y de beneficio general, sin embargo, en todos los casos no lo es, no por sus resultados o tiempo gastado en la recolección de datos que resulta de una investigación, sino por el manejo no ético con el que se ha llevado la búsqueda de información, lo que a veces puede recaer en investigaciones vagas, dañinas o sin productividad alguna, esta situación perjudica a la sociedad en general, es por ello que en este artículo científico se demostrará la importancia de la personalidad ética en la recolección de información de una investigación, sabiendo que de esa forma se forman grandes profesionales y no lo contrario.

En los últimos años se ha presentado un evidente aumento de duplicas de contenidos de los autores en las revistas científicas y esto es originado por la presión que tienen los 
investigadores en publicar dentro del tiempo popular, esto ha ocasionado múltiples modos y formas de investigación antiéticas ejercidas por los nuevos investigadores científicos. Se debe dar a conocer a aquellos que desean hacer de la investigación su vida, sobre lo relevante que implica realizar un trabajo bajo el ojo crítico de la Ética, ya que si se siguen aplicando facilismos para el plagio y la duplicación no estaríamos hablando de Ciencia sino únicamente de contenido regenerado, reutilizado y sobre todo ilegal, Caso contrario con este comportamiento no se está haciendo ciencia sino información reciclada ilegalmente.

\section{Materiales y Métodos}

El tipo de investigación es dogmática con enfoque cualitativo, el alcance de la investigación es descriptivo explicativo.

Con el enfoque cualitativo se tiene una gran amplitud de ideas e interpretaciones que enriquecen el fin de la investigación. El alcance final del estudio cualitativo consiste en comprender un fenómeno social complejo, más allá de medir las variables involucradas, se busca entenderlo (Sampieri, 2006, pp. 3-26).

Se utiliza de igual manera el método descriptivo explicativo para este trabajo, ya que es considerado uno de los métodos y modos de carácter cualitativo que se usan en investigaciones que tienen como objetivo la evaluación de algunas características de una situación en particular. En la investigación descriptiva, el objetivo es describir el comportamiento o estado de un número de variables. El método descriptivo orienta al investigador en el método científico (DIARIO, 2018).

En este proceso de investigación se utilizará el método analítico, el cual se basa en el estudio de las partes del todo y establecer los nexos. Según Hobbes, el método analítico parte de lo conocido, del efecto, se centra en el estudio de las partes y las causas de las mismas. Por lo que se entenderá el todo compuesto por todos los elementos y la relación que existe entre ellos. Para ello, se acudirá a la revisión bibliográfica de base de datos, libros y artículos académicos relevantes sobre el objeto de estudio.

Para establecer el orden y razonamiento en el presente artículo se utilizará el método inductivo-deductivo, que conduce hacia el razonamiento; método que permite pasar hacia los principios generales desde hechos particulares. "Al analizar estos hechos se encontrarán las relaciones entre las mismos, lo que conduce hacia una teoría inferida 
inductivamente, pues se encuentra dentro de las premisas" (Hurtado León \& Toro Garrido).

Y para finalizar se usará el método hipotético demostrativo. Según Aristóteles, se conoce como demostración al silogismo que produce ciencia; entonces, el principio lógico consiste en la derivación, y ésta se basa en analogías, metáforas para lograr sus cometidas, es decir la verdadera transferencia de las premisas hacia las conclusiones. Con este método se logrará demostrar los resultados de los silogismos específicos de la finalidad de este artículo.

\section{Resultados}

Para el desarrollo y discusión del presente tema vamos a considerar tres fases, la primera es la Investigación, la segunda la Ética y, por último, la tercera que será la reflexión sobre su importancia en la investigación. Es así que, en primer lugar, que se ha tomado en cuenta las posiciones de varios autores referente a lo que consideran por Investigación con fines científicos y profesionales:

En un primer momento tomamos en consideración el punto de vista que mantiene Altuve y Rivas (1998) acerca de la investigación y su proceso "la investigación es una estrategia general que adopta el investigador como forma de abordar un problema determinado, que permite identificar los pasos que deben seguir para efectuar su estudio" (p. 231).

En cuanto a la etimología de la Investigación se considera que proviene del latín "in" (en), vestigare (hallar, indagar). "Es un proceso, desarrollo o sucesión de con aspectos de reflexión, órdenes sistemáticos y examinado, sobre todo analítico y que su propósito es encontrar e inspeccionar hechos, fenómenos y leyes" (Ezequiel Ander-Egg, 1998, p.57).

Complementando a esta definición Vyhmeister (1998) en relación al verbo rector de la palabra investigación refiere lo siguiente:

La investigación busca la verdad, toda la verdad de Dios, porque Dios es la verdad, y toda la verdad proviene de él, ya sea historia, ciencia o teología. Dios es la verdad última, y los seres humanos tienen un límite que nos les permite llegar a más, por lo tanto, las conclusiones sobre la verdad no pueden considerarse definitivas. La realidad de hoy puede cambiar mañana debido a nuevos descubrimientos. Esto lleva a las personas a reconocer la necesidad de permanecer humildes y reconocer que siempre habrá cosas nuevas que aprender e investigar (pp.89-95). 
De acuerdo a lo citado se podría asegurar que un investigador es alguien en el mundo que se encarga de cierta actividad de averiguación y exploración, independiente del método, finalidad y significación. Se dice que el humano guarda una inclinación innata a encontrar el concepto y sentido de todo lo que ve, toca y siente, desde pequeño, pregunta al mayor, y ya en la juventud se va admirando.

De aquello se desprende que hay infinidad de clases de investigaciones, es decir, podemos partir desde lo más ordinario y común hasta una investigación superior o científica con ciertos parámetros y metodologías. Para Tamayo (1994) "La investigación es un proceso que, mediante la aplicación del método científico, intenta recopilar contenido importante y confiable con fines de entendimiento, verificación, corrección y aplicación del conocimiento basto." (p.45)

Es necesario saber que la investigación científica no es una exploración o averiguación que se puede hacer en cualquier momento, lugar o, de cualquier manera, sino que "es una investigación crítica, controlada y empírica de rarezas, fenómenos o milagros, dirigida por la ideología y la suposición o presunción en relación a los interrogados lazos existentes entre esas rarezas o milagros. (Kerlinger, 1983, p.11). La indagación de información y certezas que den paso a desarrollar, generalizar, explicar y predecir las rarezas que se encuentran o aparecen en el medio natural y en la colectividad humana; es una etapa singularizada de la metodología en el campo científico.” (Zorrilla \& Torres, 1994, p.29)

Por lo expuesto y manifestado, se puede asever que la investigación como tal está enfatizada y encaminada por la recolección de datos y contenido o la búsqueda de remedios para algunos males y problemas sociales, es así que, de acuerdo a Pérez y Gardey (2012):

Cabe destacar que cuando hablamos de investigación, sobre todo en el ámbito científico, es un desarrollo sistemático y organizado, esto quiere decir que se recluta información partiendo de una idea previa que, se encuentra ya aprobada y reconocida, cambiará o complementará datos e información a los ya encontrados, organizado, es de vital importancia puntualizar los mínimos detalles y rasgos relacionados a la investigación y finalidades, sus comentarios concluyentes no se respaldan en el sentido subjetivo de la palabra, sino en temporadas y situaciones que anteriormente han sido vistas y evaluadas.

Por ejemplo, lo que hacemos en estos momentos es desarrollar una investigación de carácter científica sobre la importancia y relevancia de la ética y sus principios en la investigación, es por ello que se ha tenido que seguir parámetros y directrices con la finalidad de recolectar la 
mejor información y consecuentemente respetar el criterio de cada autor, es por ello que Wesley (1982) manifiesta al respecto que:

El procedimiento que conlleva un gran aspecto formal, organizado y atareado de llevar a cabo métodos de análisis científicos tiene como objetivo descubrir actividades más sistemáticas para el desarrollo de sistemas de conocimiento organizados. Se basa en un análisis crítico de proposiciones hipotéticas, el propósito es establecer una relación causal, que debe ser probada contra la realidad objetiva. Este propósito puede ser expresar teorías o teorías de aplicación para que los eventos causados por operaciones o causas específicas puedan predecirse y finalmente controlarse (pp. 25,26).

Con todo lo aportado hasta este momento podemos concluir que una investigación conlleva mucho trabajo y tiempo para obtener excelentes resultados, no es algo que se hace de la noche a la mañana, es por ello que debe ser tratado con el respeto debido y la ética correspondiente, consecuentemente, en lo que respecta a la ética podemos decir que mucho más allá de ser una moda o un símbolo de pureza y no maleficencia, la ética y los valores que conlleva se ha convertido en una característica fundamental que se debe aplicar en la educación actual ya sea a nivel de secundaria, tercer nivel e incluso cuarto nivel, en todos los ámbitos, en el Derecho, en Administración de Empresas, en la Medicina, en sí en todos los campos que generan beneficio a la sociedad.

Científicamente la investigación en esta modalidad se considera un progreso histórico, sin embargo, debemos reflexionar si estamos investigando de manera consciente, teniendo en cuenta que es lo que puede dañar y deteriorar una simulada investigación, sin conllevar una verdadera ética ni valores en su desarrollo, sobre todo cuando se tenga que aplicar en la vida social, que repercusión tendrá.

La ética en su totalidad se considera uno de los campos más relevantes de la filosofía. Está íntimamente relacionado con conceptos como la moral, que se considera sus sinónimos, valores y cultura, y se destaca al tomar decisiones porque "está relacionado con la conciencia humana y el comportamiento responsable". (Gurria, 1996, p.37); Del mismo modo, la ética, un término estrechamente relacionado con la virtud, la moral y el carácter, aparece como un recurso o elemento (incluso si no es el más importante). Esta es una relación que uno debe desarrollar y lograr la excelencia y la armonía. La influencia de la filosofía griega siempre ha sido el fundamento básico de las creencias y los patrones de comportamiento de la cultura occidental (Cuneo, 1999, p.32). 
Tengamos en cuenta que la ética, guarda principios y reglas que no necesariamente se encuentra en una norma legal para que sean aplicadas, sino que la misma sociedad la impone, y será a criterio y humanidad de cada persona acatar esas medidas en la investigación y en su vida diaria, es por ello que Taverner, Gascó y Cortés (1997) consideran que:

La ética es la filosofía que reflexiona sobre la moral, y pretende legitimarla, o deslegitimarla, a partir de unos principios éticos. Éstos últimos son universales, ya que deben ser compartidos y respetados por cualquier individuo, independientemente de sus valores, de su moral. De ahí que también se define a la ética como el conjunto de principios y reglas morales que regulan el comportamiento y las relaciones humanas (pp. 175-187).

\section{Discusión}

Desde un inicio, en la historia del desarrollo de la sociedad, la ética ha mantenido importancia para muchos filósofos, para los educadores, y para los pensadores, quienes pensaron, inculcaron y repartían sus ideas, pensamientos y las conclusiones con discípulos designados de tal modo en ese tiempo, al igual con las sociedades y la colectividad, incluido las autoridades y gobernadores que desearon escucharlos, igualmente con otras muchas personas. Partiendo de aquello se quiso que siempre se inmortalice su voz, para poder ser escuchados hasta la actualidad, y eso se ha llegado a obtener mediante la aplicación de una conducta ética que respeta los pensamientos que nacen de otras personas sin hacerlas suyas. Es por ello que respecto a la moral Camps (1992) comenta que:

Como estudio de la moral, en primer lugar, la moral es una filosofía de práctica cuya misión no es resolver los conflictos con precisión, sino resolverlos. La teoría de la justicia y la ética de la comunicación no han señalado su supuesto camino seguro hacia la "sociedad ordenada" o la "comunidad de diálogo ideal". Es el largo viaje que debemos emprender lo que requiere una reflexión moral urgente y continua (p.27).

Respecto a lo citado cabe reconocer que se necesita de manera urgente unir la educación con los principios éticos y correlacionarlos con todas las materias del conocimiento, investigación y aprendizaje, incluyendo en los dominios de la sociedad, del campo laboral y de la vida, de manera tal que nos enriquezca a todos los seres humanos y a la naturaleza, tanto en el plano material o físico como en el mental y espiritual, por lo expuesto es que Mercader (2006) reafirma que "el objetivo innato se trata o versa en alimentar la personalidad y los valores con bases morales de los estudiantes desde temprana edad" (Mercader, 2006, pp.48,53). 
Por otra parte, filosos, políticos y pensadores consideran que una conducta ética es responsabilidad únicamente de los padres en el hogar y de los gremios religiosos, lo cual les justifica que los conceptos y contenidos fundamentales acerca de principios morales y éticos no sean impartidos ni socializados en entidades con fines educativos como tienen que realizarlo. Fisher (2003):

Insistió en que adoptar una actitud superficial o evasiva hacia la moral no promovería fundamentalmente el comportamiento moral, y que las actitudes profundas motivadas por el deseo de hacer lo correcto y mantener una vida buena o moral probablemente marcarían una gran diferencia (p.33).

Por lo expuesto, por motivos de la presente investigación, la definición de ética más adecuada y que es competente para resumir y esclarecer las definiciones y conceptualizaciones anteriores, es aquella originada y divulgada por Connock y Johns (1995), establecen que:

Hablar de moralidad es hablar de justicia, tomar decisiones entre lo correcto y lo incorrecto, y definir cómo aplicar reglas para promover un comportamiento responsable en individuos y grupos. Esta es también la esencia de todos, y en el fondo de nuestros valores, afecta las decisiones de todos (p.10).

Llegando a este punto, citaremos situaciones y consideraciones que aportan a la tesis de "importancia y relevancia de la ética y sus principios en la investigación científica que se sostiene", en primer lugar, tenemos un ejemplo de un caso en particular presentadas en España, que es el resultado de una investigación igualmente y es el siguiente:

Los autores de una encuesta investigaron el comportamiento académico deshonesto de estudiantes universitarios en el examen y desarrollo de trabajos académicos. Primero, copian durante el examen, costillas (notas que se copiarán durante el examen), se auto-replican, reemplazan al compañero en el examen, usan el teléfono móvil para obtener una copia del examen por adelantado, etc. Puede usar y copiar clips o completar documentos en segundos sin tener que proporcionar fuentes en documentos impresos y en línea. Descubrieron que cinco de cada diez jóvenes plagiaban a sus parejas o preparaban hojas de trucos. Destacaron que esto no es exclusivo de los estudiantes españoles, ya que otros estudios han reconocido situaciones similares entre los estudiantes de países como Estados Unidos, Rusia, Holanda, Moldavia, Bulgaria y China (Sureda \& Comas, 2009).

Del mismo modo en Colombia, Díaz, González y Carmona (2010):

Descubrieron que el fraude académico es común en los medios universitarios, lo que tiene un impacto negativo en la capacitación disciplinaria y los valores profesionales esenciales para el 
mercado laboral. El autor encuestó a estudiantes en Cartagena, Colombia. Reconocen la heterogeneidad de los estudiantes de odontología en la Universidad de Cartagena porque provienen de diferentes regiones geográficas y sus condiciones religiosas, socioeconómicas y culturales. Creen que "la razón de su fraude académico se relaciona con circunstancias personales que se derivan o se relacionan con sus características demográficas específicas o ambiente persistente ... con requisitos académicos obvios. (p.87)

Estos casos son reales y aquí es en donde la ética no está interviniendo, se están formando bachilleres y profesionales irresponsables y sin compromiso alguno con la sociedad, ahí es cuando recalcamos la importancia de la ética en la investigación, ya que hasta para hacer un trabajo de dos líneas hay que hacerlo de la manera correcta. Por otro lado, acotando a esto, debemos reconocer que la tecnología, así como ha aportado inmensamente en el tema de investigación científica también ha creado un aspecto negativo y estas son las mil maneras de plagio que facilitan un trabajo de investigación.

Recalcando la incidencia que tiene la ética en la investigación nos encontramos con este tercer y último ejemplo, que se debería tener siempre en cuenta antes de trabajar sin ética alguna; es el caso de Paolo Macchiarini, un médico cirujano que lanzó su fama por un fingido avance en el campo de la medicina que supuestamente aseguraba modificar y mejorar la experiencia y resultados del trasplante de órganos. El científico italiano utilizó un andamio sintético esparcido con células madre del paciente para el trasplante traqueal. Sin embargo, resulta que sus experimentos humanos no tienen una base sólida de investigación preclínica. Al menos 7 de los 9 pacientes que recibieron tratamiento murieron.

Múltiples encuestas han demostrado que el cirujano hizo uso fraudulento de algunos de los datos en sus publicaciones e informes científicos, omitiendo o incluso inventando los resultados y conclusiones para que su tratamiento parezca más exitoso y famoso. Las decisiones que rodean todas las acciones también han sido severamente criticadas. $\mathrm{Al}$ mismo tiempo, el escándalo provocó el despido de Machiarini, y el Instituto Karolinska sueco (el antiguo empleador de Machiarini) renunció. Varios documentos escritos por cirujanos italianos también han llamado la atención, incluidos dos artículos muy citados publicados en Nature Communications y The Lancet (Academy, 2020).

Para finalizar, esta parte he creído citar las palabras de Sócrates (470 - 399 AC):

Aseguró que el propósito de nuestro conocimiento debería ser aprender una vida mejor y vivir mejor bajo la premisa de la corrección moral. Sócrates enfatizó que cuando las personas hacen 
cosas malas, siempre están por ignorancia, lo que confirma que la educación moral es el objetivo de la educación (Danto, 1997, p.32).

\section{Conclusiones}

Por todo lo expuesto en este trabajo, podemos asegurar que un aprendizaje de ética puede transformar la información desde una simple difusión a aprendizaje significativo para la autonomía y el trabajo en grupo, logrando así el objetivo de mejorar el crecimiento personal y la convivencia. De conformidad con los derechos y la buena vida establecidos en la Constitución, la educación se lleva a cabo de esta manera, y esto se utiliza para promover el desarrollo de todo el conglomerado social.

Igualmente, después de una ardua revisión bibliográfica se puede concluir que una conducta ética es importante en el campo educativo y científico, con el establecimiento de esta, se crearía bases de confianza y buena fe de todos los que nos interesamos en investigar en pro de la sociedad, por otro lado, consideremos que la existencia de normas sancionadoras no debería ser motivo para ajustarnos a la ética, ya no se trata de leyes sino de comportamiento, para tener una buena ética profesional.

Por otro lado, por experiencia, en esta etapa de educación superior, se debería implementar talleres a conciencia de lo que implica una mala investigación, y lo que nos espera en un futuro profesional, en donde la deshonestidad no será recompensada, sino mal vista por la sociedad. Finalmente, podemos decir que todo lo citado cumple un rol especial, la investigación definida nos ha dado una noción de que campo es el que vamos a abarcar, el estudio de la ética nos hizo concebirla como una herramienta vital, no únicamente en lo académico sino en la vida general y por último, hemos llegado a demostrar que debe existir una verdadera conducta y actividad con principios totalmente éticos en las investigaciones que a futuro se tendrán que desarrollar y realizar, de esa forma se crea y origina respeto por nuestros trabajos y el de los demás en cualquier campo social económico y cultural.

\section{Referencias}

Academy, E. (17 de enero de 2020). Enago Academy. Recuperado el 28 de mayo de 2020, de https://www.enago.com/es/academy/importance-of-research-ethics/\#disqus_thread

Altuve, S., \& Rivas, A. (1998). Metodología de la Investigación. Módulo Instruccional 111. Caracas: Universidad Experimental Simón Rodríguez. 
Best, W. (1982). ¿Como investigar una educación? Madrid España: Ediciones Morata S.A.

Camps, V. (1992). Concepciones de la Ética. Madrid: Editorial Trotta.

Conock, S., \& Johns, T. (1995). Ethical Leadership. London: Institute of personnel and Development.

Cuneo, S. (1999). Virtudes. Barcelona España: Ediciones B.S.A.

Danto, E. (1997). Como educar en valores. España: B.S.A. editores.

DIARIO, O. (21 de junio de 2018). QUE ES EL METODO DESCRIPTIVO. La investigación, importante en el método descriptivo, pág. s/n.

Diaz, A., González, F., \& Carmona, L. (26 de febrero de 2010). S cielo. Recuperado el 28 de mayo de 2020, de Relación del fraude académico con situaciones personales que enfrentan los jóvenes en la Facultad de Ciencias Odontológicas de la Universidad de Cartagena: http://www.scielo.org.co/pdf/sun/v26n1/v26n1a1o.pdf

Ezequiel Ander-Egg. (1998). Introducción a la Técnicas de Investigación Social. Buenos Aires: Humanista.

Fisher, R. (2003). Como educar en valores. España: Obelisco.

Gurria, M. (1996). Ética Profesional: El turismo como ejemplo. México: Universidad de las Américas Puebla.

Hurtado León, I., \& Toro Garrido, J. (s.f.). Paradigmas y métodos de investigación en tiempos de cambios. (E. Nacional, Ed.) Recuperado el 17 de 03 de 2019, de https://books.google.com.ec/books?id=pTHLXXMa9osC\&pg=PA65\&dq=metodo+analitico + de+investigaci\%C3\%B3n\&hl=es\&sa=X\&ved=oahUKEwj7zLyn5aXhAhVHjlkKHYbrD4oQ6 $\mathrm{AEILjAB} \# \mathrm{v}=$ onepage $\& \mathrm{q}=$ inductivo\&f$=$ false

Kerlinger, F. (1983). Investigación del Comportamiento. Técnicas y Metodología. México: Ed. Interamericana.

Mercader, V. (2006). A study of ethical values of college students. Florida-USA: University of South.

Perez, J., \& Gardey, A. (s/f de s/f de 2012). Definición de. Obtenido de https://definicion.de/investigacion/

Sampieri, R. (2006). Metodología de la Investigación. México: Mc Graw-Hill. 
Sureda, J., \& Comas, R. (s/f de julio de 2009). Dadun. Recuperado el 28 de mayo de 2020, de Practicas académicas deshonestas en el desarrollo de exámenes: http://dadun.unav.edu/bitstream/10171/9852/2/17Ef.pdf

Tamayo, M. (1994). El progreso de la Investigación Científica. México: LIMUSA Noriega Editores.

Taverner, J. L., Gascó, J. L., \& Cortés, E. C. (1997). ETICA EMPRESARIAL: implicaciones para la dirección de los recursos humanos. España: Boletín de Estudios Económicos.

Whymeister, N. (1998). Women in Ministry: Biblical and Historical Perspectives. USA: Andrews University Press.

Zorrilla, S., \& Torres, M. (1994). LA TESIS SEGUNDA EDICION. México: Ed. McGraw-Hill. 